\title{
Expression of proliferating cell nuclear antigen (PCNA) in gastric carcinoma: no evidence for prognostic value
}

\author{
D C Mangham, D C Rowlands, K M Newbold, G M Reynolds, J W L Fielding, \\ M T Hallissey
}

\begin{abstract}
It has been proposed that immunostaining with PC10, a monoclonal antibody against proliferating cell nuclear antigen (PCNA), is of prognostic value in gastric carcinoma. Gastric carcinomas from a series of 90 patients in whom survival data were known have been studied. There was no relation between the degree of PC10 immunostaining assessed semiquantitatively and survival.
\end{abstract}

(F Clin Pathol 1994;47:473-474)

The monoclonal antibody $\mathrm{PC} 10$ recognises proliferating cell nuclear antigen (PCNA) in sections of formaldehyde fixed paraffin embedded tissue. ${ }^{12}$ The expression of PCNA seems to be deregulated in many types of tumour, including gastric carcinoma, and so may overestimate the growth fraction..$^{2-6}$ Several studies have suggested, however, that there is a relation between the proportion of tumour cells positive for PCNA and prognosis, ${ }^{78}$ including two studies that have considered gastric cancer. ${ }^{69}$ Our study reports the expression of PCNA in a series of 90 patients with gastric adenocarcinoma on whom clinical follow up data were available.

\section{Methods}

Sections $(4 \mu \mathrm{m})$ from representative tumour blocks of 90 gastric cancers were immunostained with PC10 (M 879, Dako UK) for 30 minutes at a dilution of 1 in 25 (an antibody concentration of $23 \mu \mathrm{m} / \mathrm{ml}$ ). An immunoperoxidase detection system was used with a biotinylated rabbit anti-mouse immunoglobulin secondary (PB270, Binding Site, Birmingham, England) diluted 1 in 100, followed by peroxidase conjugated streptavidin (IC019, Binding Site) at a dilution of 1 in 200. Sections from each case were also stained with RET 40f (M 820, Dako), an antibody against glycophorin $\mathrm{C}$, as a negative control. The sections were visualised with diaminobenzidine and counterstained with haematoxylin. The stained sections were assessed independently by three observers (DCM, KMN, and DCR). The semiquantitative grading system that had been found to be the most discriminative in a previous study into gastric carcinoma was used. ${ }^{6}$ Based on an examination of the whole section a subjective assessment of the proportion of tumour cell nuclei that showed definite immunopositivity was made. Tumours were divided into two grades, representing the proportion of tumour cells positive with $\mathrm{PC} 10$ as less than or greater than $50 \%$. Survival was analysed with the log rank test and the relation between PCNA grade and other clinicopathological variables was assessed with the McNemar test.

\section{Results}

The tumours studied included 12 early and 78 advanced gastric cancers. The ratio of men to women was $1 \cdot 8: 1$. The median age was 68 (range 35 to 87 ) years. The minimum follow up period was three years, median eight years.

There was good staining of erythrocytes with RET 40f, but no nuclear staining was seen with this antibody. Strong immunostaining with PC10 was found in a proportion of tumour cell nuclei in every case included in this study. In those sections that included normal gastric mucosa, $\mathrm{PC} 10$ positivity in the epithelium was limited to the proliferating zone and no staining was seen in superficial epithelial cells or within the specialised glands.

The consensus between the three observers for the PCNA grade was $88 \% \quad(\kappa=0 \cdot 81)$. Specimens for which there was disagreement were reassessed and all assigned to the grade chosen by the majority of observers. Thirty

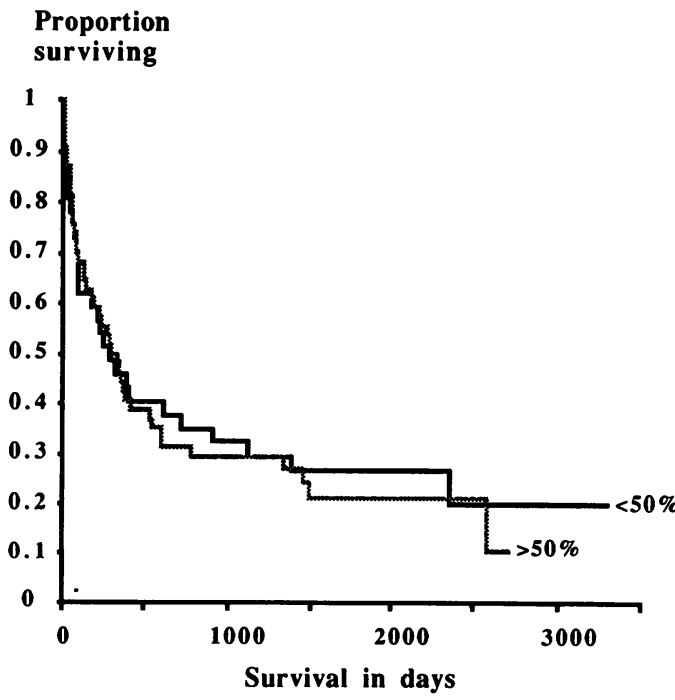

Survival curves of patients with high and low levels of PCNA expression. There is no survival difference between those patients having high and low proportions of PC10 positive tumour cells. 
seven of the tumours were categorised in the less than $50 \%$ grade and 53 tumours as having greater than $50 \%$ of tumour cells positive. There was no significant difference between the survival curves for these two groups (figure). When the tumours were graded into four categories, corresponding to $1-25 \%$, $26-50 \%, 51-75 \%$ and $76-100 \%$ of tumour cells positive with PC10, all four survival curves overlapped. Thus it is considered unlikely that any relation between PC10 staining and survival would be found in this series if the division between high and low grades were set at a different level. Observer agreement by the four grade system was poor, however, $(\kappa=0.49)$. There was no relation between PC10 grade and sex, age, histological type (Lauren), tumour stage, or lymph node state.

\section{Discussion}

The results of this study do not support the suggestion that $\mathrm{PC} 10$ grading has prognostic value in gastric carcinoma. This contrasts with the previous studies, ${ }^{69}$ and may be due to differences between the groups. The five year survival of the patients in both the previous studies was much better than in the present series, which showed a survival pattern more typical of gastric carcinoma. This is despite the fact that Jain et $a l^{6}$ studied only advanced gastric cancers. In the present study more tumours had high PC10 grades than was described by the other two papers, which may also reflect a difference between the groups of cases. This is more likely, however, to be a reflection of the difficulties encountered in standardising PC10 immunostaining. In particular, the type and duration of tissue fixation, preparation of sections, and differences in the immunostaining protocols can have a large effect upon the proportion of cells that are labelled with this antibody. ${ }^{2410}$ Also, there are problems associated with the pronounced heterogeneity within these tumours, such that any one tumour block may not be representative. In this study the subjective grading of the tumours was difficult and reproducibility between observers was imperfect.

This study was supported by a grant from the Endowment Fund of the former United Birmingham Hospitals. We are grateful to $\mathrm{C}$ Glendining for assistance in preparing the figure.

1 Waseem NH, Lane DP. Monoclonal antibody analysis of the proliferating cell nuclear antigen (PCNA) the proliferating cell nuclear antigen (PCNA). Structural conservation and the
form. $F$ Cell Sci 1990;96:121-9.

2 Hall PA, Levison DA, Woods AL, et al. Proliferating cel nuclear antigen (PCNA) immunolocalization in paraffin sections: An index of cell proliferation with evidence of deregulated expression in some neoplasms. F Pathol 1990;162:285-94.

3 Scott RJ, Hall PA, Haldane JS, et al. A comparison of immunohistochemical markers of cell proliferation with experimentally determined growth fraction. 7 Pathol 1991;165:173-8.

4 McCormick D, Hall PA. The complexities of proliferating cell nuclear antigen. Histopathology 1992;21:591-4.

5 Rosa JC, Mendes R, Filipe MI, Morris RW. Measurement of cell proliferation in gastric carcinoma: comparative analysis of $\mathrm{Ki}-67$ and proliferative cell nuclear antigen (PCNA). Histochem F 1992;24:93-101.

6 Jain S, Filipe MI, Hall PA, Waseem N, Lane DP, Levison DA. Prognostic value of proliferating cell nuclear antigen in gastric carcinoma. $\mathcal{F}$ Clin Pathol 1991;44:655-9.

$7 \mathrm{Yu}$ CC-W, Hall PA, Fletcher CDM, et al. Haemangiopericytomas: the prognostic value of immunohistochemical staining with a monoclonal antibody to proliferating cell nuclear antigen (PCNA) Histopathology 1991;19:29-33.

8 Yu CC-W, Fletcher CDM, Newman PL, Goodlad JR, Burton JC, Levison DA. A comparison of proliferating cell nuclear antigen (PCNA) immunostaining, nucleola organizer region (AgNOR) staining, and histological grading in gastrointestinal stromal tumours. F Patho 1992;166:147-52.

9 Yonemura Y, Kimura H, Fushida S, et al. Analysis of proliferative activity using anti-proliferating cell nuclear antigen antibody in gastric cancer tissue specimen obtained by endoscopic biopsy. Cancer 1993;71: 2448-53.

10 McCormick D, Yu C, Hobbs C, Hall PA. The relevance of antibody concentration to the immunohistological quantification of cell proliferation-associated antigens. Histopathology 1993;22:543-47. 